\title{
BLICHFELDT-TYPE INEQUALITIES AND CENTRAL SYMMETRY
}

\author{
MARTIN HENK, MATTHIAS HENZE AND JÖRG M. WILLS
}

Dedicated to Professor Peter M. Gruber on the occasion of his 70th birthday

\begin{abstract}
A classical result of Blichfeldt, from 1921, gives a sharp lower bound on the volume of a convex body $K$, whose lattice points span the whole space, in terms of the lattice point enumerator $\#\left(K \cap \mathbb{Z}^{n}\right)$. We are interested in a version of this inequality on the set of 0 -symmetric convex bodies. Our motivation to study this problem comes from a lack of methods that exploit the symmetry assumption in problems of a similar kind and where 0 -symmetry is a natural condition. We report upon sharp Blichfeldt-type inequalities for 0-symmetric lattice polygons, lattice crosspolytopes and lattice zonotopes.
\end{abstract}

\section{INTRODUCTION}

Let $\mathcal{K}^{n}$ be the set of all convex bodies in $\mathbb{R}^{n}$, i.e., compact convex sets with non-empty interior. A body $K \in \mathcal{K}^{n}$ is called centrally symmetric if there exists an $x \in \mathbb{R}^{n}$ such that $K-x=-(K-x)$. If $x=0$ we say that $K$ is 0 -symmetric and we let $\mathcal{K}_{0}^{n}$ be the family of all 0 -symmetric convex bodies in $\mathbb{R}^{n}$. When dealing with polytopes we write $\mathcal{P}^{n}$ and $\mathcal{P}_{0}^{n}$, respectively. The family of $n$-dimensional lattices in $\mathbb{R}^{n}$ is denoted by $\mathcal{L}^{n}$ and the usual Lebesgue measure with respect to the $n$-dimensional Euclidean space by $\operatorname{vol}_{n}(\cdot)$. If the ambient space is clear from the context we omit the subscript and just write $\operatorname{vol}(\cdot)$. For a given bounded subset $S \subset \mathbb{R}^{n}$ and a lattice $\Lambda \in \mathcal{L}^{n}$ the lattice point enumerator is denoted by $\mathrm{G}(S, \Lambda)=\#(S \cap \Lambda)$. If $\Lambda=\mathbb{Z}^{n}$ we shortly write $\mathrm{G}(S)=\mathrm{G}\left(S, \mathbb{Z}^{n}\right)$. Finally, for a subset $A \subseteq \mathbb{R}^{n}$ the dimension of its affine hull will be denoted by $\operatorname{dim} A$.

The determination of bounds for the lattice point enumerator $\mathrm{G}(K)$ of a convex body $K \in \mathcal{K}^{n}$ in terms of continuous magnitudes is a classical problem which was initiated by a result of Blichfeldt [5].

2010 Mathematics Subject Classification. 52C07, 52B20, 52A40, $11 \mathrm{H} 06$.

Key words and phrases. Central symmetry, zonotope, crosspolytope, Ehrhart polynomial, lattice polytopes.

The work of the second author was supported by the Deutsche Forschungsgemeinschaft (DFG) within the project He 2272/4-1. 
Theorem 1.1 (Blichfeldt, 1921). Let $K \in \mathcal{K}^{n}$ be a convex body such that $\operatorname{dim}\left(K \cap \mathbb{Z}^{n}\right)=n$. Then

$$
\operatorname{vol}(K) \geq \frac{1}{n !}(\mathrm{G}(K)-n) .
$$

This inequality is best possible, as the simplices $S_{k}=\operatorname{conv}\left\{0, k e_{1}, e_{2}\right.$, $\left.\ldots, e_{n}\right\}$ show, where $k \in \mathbb{N}$ and $e_{i}$ denotes the $i$-th unit vector in $\mathbb{R}^{n}$. Here we have $\mathrm{G}\left(S_{k}\right)=n+k$ and $\operatorname{vol}\left(S_{k}\right)=\frac{k}{n !}$. Note, that the condition $\operatorname{dim}(K \cap$ $\left.\mathbb{Z}^{n}\right)=n$ is essential for inequality (1.1). If all lattice points of $K$ would be contained in a proper affine subspace of $\mathbb{R}^{n}$, then the volume of $K$ can be arbitrarily small. An inequality of the above type is invariant under lattice preserving transformations and furthermore $K$ can be assumed to be a polytope which is often more convenient for combinatorial arguments. Indeed, setting $P_{K}=\operatorname{conv}\left\{K \cap \mathbb{Z}^{n}\right\}$, we clearly have $\operatorname{vol}(K) \geq \operatorname{vol}\left(P_{K}\right)$ and $\mathrm{G}(K)=\mathrm{G}\left(P_{K}\right)$. Also it is no loss of generality when we restrict to the integer lattice $\mathbb{Z}^{n}$. This is because if $\Lambda=A \mathbb{Z}^{n}$ for some invertible matrix $A \in \mathbb{R}^{n \times n}$, then $\operatorname{vol}\left(A^{-1} K\right)=\frac{\operatorname{vol}(K)}{\operatorname{det}(\Lambda)}$ and $\mathrm{G}(K, \Lambda)=\mathrm{G}\left(A^{-1} K, \mathbb{Z}^{n}\right)$.

In this work we are interested in a Blichfeldt-type inequality on the set of 0 -symmetric convex bodies $K \in \mathcal{K}_{0}^{n}$, and, in particular, deal with the question whether 0 -symmetry yields an improvement of $(1.1)$ by an exponential factor in the dimension. Although 0-symmetry is a very natural condition, in many contexts with a combinatorial flavor it is not clear how to exploit it. Famous examples for this lack of methods are the so called $3^{d}$-conjecture of Kalai [10] and the upper and lower bound problem on $f$ vectors of 0 -symmetric polytopes (see [17, Lect. 8]). The only general result in this context is due to Stanley [16] who was able to solve the lower bound problem for simplicial 0-symmetric polytopes.

First of all we will be concerned with the situation in the plane. Here, the condition of central symmetry for $P \in \mathcal{P}^{2}$ is equivalent to saying that $P$ is a zonotope. This observation together with an inequality of Scott [14] lead to satisfactory answers to the planar problem. Two given bodies $K, L \in \mathcal{K}^{n}$ are called unimodularly equivalent, in symbols $K \simeq L$, if there is a latticepreserving affine transformation that maps $K$ to $L$.

Theorem 1.2. Let $P \in \mathcal{P}^{2}$ be a lattice polygon that is not unimodularly equivalent to the triangle $S^{*}=\operatorname{conv}\left\{0,3 e_{1}, 3 e_{2}\right\}$ and contains at least one interior lattice point. Then,

$$
\operatorname{vol}(P) \geq \frac{2}{3}(\mathrm{G}(P)-3) .
$$

The rectangles $R_{k}=\left\{x \in \mathbb{R}^{2}:\left|x_{1}\right| \leq 1,0 \leq x_{2} \leq k\right\}, k \geq 2$, attain equality.

Note that for even values of $k \in \mathbb{N}$ the rectangles $R_{k}$ are centrally symmetric with respect to a lattice point and therefore the above inequality is sharp on the class $\mathcal{K}_{0}^{2}$. Furthermore, for the excluded triangle $S^{*}$ we have $\operatorname{vol}\left(S^{*}\right)=\frac{9}{14}\left(\mathrm{G}\left(S^{*}\right)-3\right)$. 
Similar in flavor to results of Bárány [1] we can say considerably more and quantify the intuition that if a lattice polygon has a lot of vertices then its volume is well-approximated by its number of lattice points.

Proposition 1.1. Let $P \in \mathcal{P}^{2}$ be a centrally symmetric lattice polygon with $2 m$ vertices. Then,

$$
\operatorname{vol}(P) \geq \frac{m}{m+2}(\mathrm{G}(P)-2) .
$$

The rectangles $\left\{x \in \mathbb{R}^{2}: 0 \leq x_{1} \leq 1,0 \leq x_{2} \leq k\right\}, k \in \mathbb{N}$, attain equality.

Any 0-symmetric lattice polytope clearly contains an interior lattice point. Ehrhart theory provides tools that imply a Blichfeldt-type inequality for any lattice polytope with this property. In particular, the inequality below, which was already noted in [7, Cor. 3.3], shows that the existence of interior lattice points already gives an improvement on Blichfeldt's inequality by a factor linear in the dimension.

Proposition 1.2. Let $P \in \mathcal{P}^{n}$ be a lattice polytope with at least one interior lattice point. Then,

$$
\operatorname{vol}(P) \geq \frac{(n-1)(\mathrm{G}(P)-(n+1))+2}{n !} .
$$

Equality holds, for instance, for the simplex $\operatorname{conv}\left\{e_{1}, \ldots, e_{n},-\left(e_{1}+\ldots+e_{n}\right)\right\}$.

As mentioned earlier, we suspect that 0 -symmetry implies an exponential improvement upon (1.1). In support of this conjecture we study lattice crosspolytopes $C$ which are minimal convex bodies in the sense that any 0-symmetric convex body $K \in \mathcal{K}_{0}^{n}$ with $\operatorname{dim}\left(K \cap \mathbb{Z}^{n}\right)=n$ contains a lattice crosspolytope. An analogous result where $\mathrm{G}(C)$ is replaced by $\mathrm{G}(\operatorname{int} C)$, i.e., the number of interior lattice points of $C$, was obtained by Bey, Henk and Wills [4, Prop. 1.4]. Their argument uses techniques from Ehrhart theory which do not seem to be applicable in the situation of $\mathrm{G}(C)$ and thus our approach is a different one.

Theorem 1.3. Let $C \in \mathcal{P}_{0}^{n}$ be a lattice crosspolytope. Then

$$
\operatorname{vol}(C) \geq \frac{2^{n-2}}{n !}(\mathrm{G}(C)-(2 n-3)) .
$$

The standard crosspolytope $C_{n}^{\star}=\operatorname{conv}\left\{ \pm e_{1}, \ldots, \pm e_{n}\right\}$ shows that the inequality is tight.

The classical Blichfeldt inequality (1.1) is one example among a variety of problems that can be solved by the concept of subdivisions or, more specifically, triangulations, which often allow a local analysis and the reduction of the problem to much more handsome bodies, like simplices. In general, a 0-symmetric polytope cannot be divided into smaller centrally symmetric pieces and such an approach has to fail. On the positive side there is the important class of zonotopes that admit such a subdivision into parallelepipeds. A zonotope $Z \in \mathcal{P}^{n}$ is the Minkowski sum of finitely many line 
segments, that is, there are points $v_{1}, w_{1}, \ldots, v_{m}, w_{m} \in \mathbb{R}^{n}, m \geq n$, such that $Z=\sum_{i=1}^{m}\left[v_{i}, w_{i}\right] . Z$ is centrally symmetric with respect to the point $\sum_{i=1}^{m} \frac{1}{2}\left(w_{i}-v_{i}\right)$ and considering lattice zonotopes means that up to a lattice translation of $Z$ the $v_{i}$ 's and $w_{i}$ 's can be chosen to be lattice points. For more details on zonotopes consult for example [15, 17]. Our next theorem gives a sharp Blichfeldt-type inequality for lattice zonotopes and shows how even stronger symmetry conditions can be handled for these special bodies.

Theorem 1.4. Let $Z \in \mathcal{P}^{n}$ be a lattice zonotope.

i) Then

$$
\operatorname{vol}(Z) \geq\left(\frac{1}{2}\right)^{n-1}\left(\mathrm{G}(Z)-2^{n-1}\right)
$$

and equality holds if and only if $Z \simeq \sum_{i=1}^{n-1}\left[0, e_{i}\right]+\left[0, k e_{n}\right], k \in \mathbb{N}$.

ii) If $Z=\sum_{i=1}^{m}\left[-a_{i}, a_{i}\right]$, for some $a_{i} \in \mathbb{Z}^{n}$, then

$$
\begin{aligned}
& \qquad \operatorname{vol}(Z) \geq\left(\frac{2}{3}\right)^{n-1}\left(\mathrm{G}(Z)-3^{n-1}\right), \\
& \text { and equality holds if and only if } Z \simeq \sum_{i=1}^{n-1}\left[-e_{i}, e_{i}\right]+\left[-k e_{n}, k e_{n}\right], k \in \mathbb{N} .
\end{aligned}
$$

When we compare the inequalities in Theorems 1.1, 1.2 and 1.4 it is reasonable to conjecture that a Blichfeldt-type inequality for all $K \in \mathcal{K}_{0}^{n}$ with $\operatorname{dim}\left(K \cap \mathbb{Z}^{n}\right)=n$ looks like

$$
\operatorname{vol}(K) \geq c_{n} \cdot(\mathrm{G}(K)-(2 n-1)),
$$

where $c_{n} \geq \frac{c^{n}}{n !}$ for some absolute constant $c>1$. The additive constant on the right hand side should be the minimal number of lattice points contained in an $(n-1)$-dimensional body from the considered class. A search for examples with a constant $c_{n}$ in 1.3 as small as possible showed that, surprisingly, lattice crosspolytopes are not the extremal bodies. But still, the constants are exponentially bigger than in Blichfeldt's inequality.

Indeed, let $C_{3}=[-1,1]^{3}$ and $Q_{n}=\operatorname{conv}\left\{C_{3}, \pm e_{4}, \ldots, \pm e_{n}\right\}$. Then, for $n \geq 3$, we have $\operatorname{vol}\left(Q_{n}\right)=3 \cdot \frac{2^{n+1}}{n !}$ and $\mathrm{G}\left(Q_{n}\right)=2 n+21$, and thus

$$
\operatorname{vol}\left(Q_{n}\right)=\frac{3}{11} \cdot \frac{2^{n}}{n !}\left(\mathrm{G}\left(Q_{n}\right)-(2 n-1)\right) .
$$

The factor $\frac{3}{11} \cdot \frac{2^{n}}{n !}$ is the smallest that we found in dimensions $3 \leq n \leq 5$. For $n \geq 6$ our best examples are $P_{n, k}=C_{n-1}^{\star} \times\left[-k e_{n}, k e_{n}\right]$, where $C_{n}^{\star}$ is the standard crosspolytope of dimension $n$ and $k \in \mathbb{N}$. Then $\operatorname{vol}\left(P_{n, k}\right)=\frac{2^{n}}{(n-1) !} k$ and $\mathrm{G}\left(P_{n, k}\right)=(2 k+1)(2 n-1)$, and therefore

$$
\operatorname{vol}\left(P_{n, k}\right)=\frac{2^{n-1}}{(n-1) !(2 n-1)}\left(\mathrm{G}\left(P_{n, k}\right)-(2 n-1)\right) .
$$


The remainder of the paper gives the details for the aforementioned results. Section 2 deals with the proof of Proposition 1.2 and the planar situation. In Sections 3 and 4 the proofs for the Blichfeldt-type inequalities for lattice crosspolytopes and lattice zonotopes are given.

\section{Proof of Proposition 1.2 And the Planar Situation}

We start with the proof of Proposition 1.2 which already can be found in [7] but is given here for the sake of completeness. To this end, we need to recall a small amount of Ehrhart theory; for details on this subject we refer the reader, for instance, to [3] and [4]. The Ehrhart polynomial of a lattice polytope $P \in \mathcal{P}^{n}$ is given by $\mathrm{G}(k P)=\sum_{i=0}^{n} \mathrm{a}_{i}(P)\left(\begin{array}{c}k+n-i \\ n\end{array}\right), k \in \mathbb{N}$, where the coefficients $\mathrm{a}_{i}(P)$ only depend on $P$. In general these coefficients satisfy $\mathrm{a}_{0}(P)=1, \mathrm{a}_{1}(P)=\mathrm{G}(P)-(n+1), \mathrm{a}_{n}(P)=\mathrm{G}($ int $P)$ and $\mathrm{a}_{0}(P)+\mathrm{a}_{1}(P)+$ $\cdots+\mathrm{a}_{n}(P)=n ! \operatorname{vol}(P)$. Furthermore, a result from Hibi [9] shows that $\mathrm{a}_{i}(P) \geq \mathrm{a}_{1}(P)$, for $i=1, \ldots, n-1$, if $\mathrm{G}($ int $P) \geq 1$. A simple combination of these relations now gives

$$
\begin{aligned}
n ! \operatorname{vol}(P) & =\sum_{i=0}^{n} \mathrm{a}_{i}(P) \geq \mathrm{a}_{n}(P)+(n-1) \mathrm{a}_{1}(P)+1 \\
& \geq(n-1)(\mathrm{G}(P)-(n+1))+2,
\end{aligned}
$$

as desired. Equality holds if and only if $\mathrm{a}_{n}(P)=\mathrm{G}($ int $P)=1$ and $\mathrm{a}_{n-1}(P)=$ $\cdots=\mathrm{a}_{1}(P)=\mathrm{G}(P)-(n+1)$.

The remainder of this section will be devoted to the planar case and the study of lattice polygons.

Proof of Theorem 1.2. This inequality is just a combination of two wellknown results for lattice polygons. The first one is an inequality by Scott [14] which states that for any lattice polygon $P$ that has at least one interior lattice point

$$
\mathrm{G}(\partial P) \leq 2 \mathrm{G}(\text { int } P)+7,
$$

and equality holds (up to unimodular equivalence) exactly for $S^{*}$. Since we want to exclude $S^{*}$, we get $2 \mathrm{G}(\operatorname{int} P) \geq \mathrm{G}(\partial P)-6$ and thus $\mathrm{G}(\operatorname{int} P) \geq$ $\frac{1}{3}(\mathrm{G}(P)-6)$.

The second result we use is Pick's Theorem [13], which states that

$$
\mathrm{G}(P)=\operatorname{vol}(P)+\frac{1}{2} \mathrm{G}(\partial P)+1 .
$$

Combining the two yields $2 \operatorname{vol}(P)=\mathrm{G}(P)+\mathrm{G}($ int $P)-2 \geq \frac{4}{3} \mathrm{G}(P)-4$, which is equivalent to the claimed inequality.

Proof of Proposition 1.1. It is well-known that centrally symmetric polygons are zonotopes (see [17, Sect. 7.3] for details). Therefore, up to a lattice translation we can write $P=\sum_{i=1}^{m}\left[0, a_{i}\right]$ for suitable pairwise non-parallel $a_{1}, \ldots, a_{m} \in \mathbb{Z}^{2} \backslash\{0\}$. A result of Shephard [15, Thm. (54)] shows that $P$ can be dissected into parallelograms that are lattice translations of $P_{i, j}=\left[0, a_{i}\right]+$ $\left[0, a_{j}\right]$, where $\{i, j\}$ runs over all two-element subsets of $[m]=\{1, \ldots, m\}$. 
Moreover, writing $\operatorname{gcd}(a)$ for the greatest common divisor of the entries of $a \in \mathbb{Z}^{2}$, we get that the number of lattice points in a half-open edge of $P$ equals $\operatorname{gcd}\left(a_{i}\right)$ for a suitable $i$. These two observations together with Pick's Theorem (see Equation (2.1) yield

$$
\begin{aligned}
\mathrm{G}(P) & =\operatorname{vol}(P)+\frac{1}{2} \mathrm{G}(\partial P)+1=\operatorname{vol}(P)+\sum_{i=1}^{m} \operatorname{gcd}\left(a_{i}\right)+1 \\
& =\sum_{\{i, j\} \in\left(\begin{array}{c}
{[m]} \\
2
\end{array}\right)} \operatorname{vol}\left(P_{i, j}\right)+\sum_{i=1}^{m} \operatorname{gcd}\left(a_{i}\right)+1
\end{aligned}
$$

The volume of a lattice parallelogram equals the number of lattice points in its half-open counterpart (see [2, p. 89]), so for any $\{i, j\} \in\left(\begin{array}{c}{[m]} \\ 2\end{array}\right)$ we have

$$
\operatorname{vol}\left(P_{i, j}\right)=\mathrm{G}\left(\left[0, a_{i}\right)+\left[0, a_{j}\right)\right) \geq \operatorname{gcd}\left(a_{i}\right)+\operatorname{gcd}\left(a_{j}\right)-1,
$$

and equality holds if and only if $\operatorname{relint}\left(P_{i, j}\right) \cap \mathbb{Z}^{2}=\emptyset$. Thus

$$
\begin{aligned}
\mathrm{G}(P) & =\sum_{\{i, j\} \in\left(\begin{array}{c}
{[m]} \\
2
\end{array}\right)} \mathrm{G}\left(\left[0, a_{i}\right)+\left[0, a_{j}\right)\right)+\sum_{i=1}^{m} \operatorname{gcd}\left(a_{i}\right)+1 \\
& \geq m \sum_{i=1}^{m} \operatorname{gcd}\left(a_{i}\right)-\left(\begin{array}{c}
m \\
2
\end{array}\right)+1=\frac{m}{2} \mathrm{G}(\partial P)-\left(\begin{array}{c}
m \\
2
\end{array}\right)+1 .
\end{aligned}
$$

Using Pick's Theorem (2.1) once more we derive

$$
\begin{aligned}
\operatorname{vol}(P) & =\mathrm{G}(P)-\frac{1}{2} \mathrm{G}(\partial P)-1 \geq \frac{m-1}{m} \mathrm{G}(P)-\frac{m^{2}+m-2}{2 m} \\
& =\frac{m-1}{m}\left(\mathrm{G}(P)-\frac{m+2}{2}\right),
\end{aligned}
$$

which is greater than or equal to the claimed $\frac{m}{m+2}(\mathrm{G}(P)-2)$ if and only if $\mathrm{G}(P) \geq 2 m+\left(\begin{array}{c}m-1 \\ 2\end{array}\right)$. This last inequality follows since the $2 m$ vertices of $P$ are lattice points and because $\mathrm{G}($ int $P) \geq\left(\begin{array}{c}m-1 \\ 2\end{array}\right)$, which we prove by induction on $m$. For $m=2$ there is nothing to show, so let $m \geq 3$ and consider $Q=\sum_{i=1}^{m-1}\left[0, a_{i}\right]$. By assumption, $Q$ has at least $\left(\begin{array}{c}m-\overline{2} \\ 2\end{array}\right)$ many interior lattice points and $2(m-1)$ vertices. No two of the $a_{i}$ 's are parallel, therefore after the addition of the remaining line segment $\left[0, a_{m}\right]$ to $Q$ exactly $m-2$ (clockwise consecutive) vertices of $Q$ are interior points of $P$ and thus $\mathrm{G}(\operatorname{int} P) \geq \mathrm{G}(\operatorname{int} Q)+m-2 \geq\left(\begin{array}{c}m-1 \\ 2\end{array}\right)$.

Recall that due to Scott [14 the inequality $\mathrm{G}(\partial P) \leq 2 \mathrm{G}($ int $P)+7$ holds for any lattice polygon $P \in \mathcal{P}^{2}$ with interior lattice points. As a generalization of Scott's result Kołodziejczyk and Olszewska [11] proved that $\mathrm{G}(\partial P) \leq 2 \mathrm{G}(\operatorname{int} P)-v(P)+10$, where $v(P)$ is the number of vertices of $P$, and proposed the problem to further improve upon this bound when $v(P)$ is large. As a corollary to Proposition 1.1 we obtain an answer to their question for centrally symmetric lattice polygons with at least six vertices. 
Corollary 2.1. Let $P \in \mathcal{P}^{2}$ be a centrally symmetric lattice polygon with $2 m \geq 6$ vertices. Then,

$$
\mathrm{G}(\partial P) \leq \frac{4}{m-1} \mathrm{G}(\operatorname{int} P)+4 .
$$

Proof. Pick's theorem 2.1) yields $\operatorname{vol}(P)=\mathrm{G}($ int $P)+\frac{1}{2} \mathrm{G}(\partial P)-1$ and together with Inequality $(2.2)$ we obtain $\mathrm{G}(\partial P) \leq \frac{2}{m-2} \mathrm{G}($ int $P)+m+1$. This is at most the claimed $\frac{4}{m-1} \mathrm{G}($ int $P)+4$ if and only if $\mathrm{G}($ int $P) \geq\left(\begin{array}{c}m-1 \\ 2\end{array}\right)$, which we have already seen in the proof of Proposition 1.1 .

\section{LAtTiCE CROSSPOLYTOPES}

Throughout this section we let $C$ be a lattice crosspolytope, that is, $C=$ $\operatorname{conv}\left\{ \pm a_{1}, \ldots, \pm a_{n}\right\}$, for some linearly independent $a_{1}, \ldots, a_{n} \in \mathbb{Z}^{n}$.

For a given $\delta \in\{-1,0,1\}^{n}$ let $\operatorname{supp}(\delta)=\left\{i \in[n]: \delta_{i} \neq 0\right\}$, consider the simplex $S_{\delta}=\operatorname{conv}\left\{0, \delta_{1} a_{1}, \ldots, \delta_{n} a_{n}\right\}$ and let $F_{\delta}$ be its facet that does not contain the origin, that is, $F_{\delta}=\operatorname{conv}\left\{\delta_{i} a_{i}: \delta_{i} \neq 0\right\}$. The simplices $S_{\delta}, \delta \in\{-1,0,1\}^{n}$, clearly define a triangulation of the crosspolytope $C$ and by definition, $S_{\delta}$ is a face of $S_{\varepsilon}$ if and only if $\operatorname{supp}(\delta) \subseteq \operatorname{supp}(\varepsilon)$ and $\delta_{i}=\varepsilon_{i}, i \in \operatorname{supp}(\delta)$. Therefore, any relative interior point of $S_{\delta}$ and $F_{\delta}$, with $\# \operatorname{supp}(\delta)=i$, is contained in exactly $2^{n-i}$ full-dimensional simplices $S_{\varepsilon}$. Let

$$
\stackrel{\circ}{S}_{\delta}=\operatorname{relint}\left(S_{\delta}\right) \cup \operatorname{relint}\left(F_{\delta}\right) \quad \text { and } \quad \mathcal{S}_{i}=\bigcup_{\substack{\delta \in\{-1,0,1\}^{n} \\ \# \operatorname{supp}(\delta)=i}} \stackrel{\circ}{S}_{\delta} \text {, for } i=0, \ldots, n .
$$

Then, $C$ is partitioned into the sets $\stackrel{\circ}{S}_{\delta}$ and thus we get $\mathrm{G}(C)=\sum_{i=0}^{n} \mathrm{G}\left(\mathcal{S}_{i}\right)$. Writing

$$
\Delta_{i}(\varepsilon)=\left\{\delta \in\{-1,0,1\}^{n}: \# \operatorname{supp}(\delta)=i, S_{\delta} \text { a face of } S_{\varepsilon}\right\},
$$

for $i=0, \ldots, n$ and $\varepsilon \in\{-1,1\}^{n}$, we have

$$
\mathrm{G}\left(\mathcal{S}_{i}\right)=\frac{1}{2^{n-i}} \sum_{\varepsilon \in\{-1,1\}^{n}} \sum_{\delta \in \Delta_{i}(\varepsilon)} \mathrm{G}\left(\stackrel{\circ}{S}_{\delta}\right) .
$$

The method of our proof of Theorem 1.3 is to attach the simplices $S_{\delta}$ to the vertices $v_{\gamma}=\sum_{i=1}^{n} \gamma_{i} a_{i}, \gamma \in\{0,1\}^{n}$, of the parallelepiped $P_{C}=\sum_{i=1}^{n}\left[0, a_{i}\right]$, apply a formula for the lattice points in $P_{C}$ and then cautiously identify lattice points in $C$ and $P_{C}$.

To make things more precise let $f: \mathbb{R}^{n} \rightarrow \mathbb{R}^{n}$ be the mapping $f_{i}(x)=$ $\frac{1-x}{2}, 1 \leq i \leq n$. Then, for all $\varepsilon \in\{-1,1\}^{n}$ we have $v_{f(\varepsilon)}+S_{\varepsilon} \subset P_{C}$. Indeed, the vertices of $v_{f(\varepsilon)}+S_{\varepsilon}$ are exactly $\sum_{j=1}^{n} \frac{1-\varepsilon_{j}}{2} a_{j}$ and $\sum_{j=1}^{n} \frac{1-\varepsilon_{j}}{2} a_{j}+$ $\varepsilon_{i} a_{i}, 1 \leq i \leq n$, which can be seen to be vertices of $P_{C}$. Thus, $\mathcal{T}(C)=$ $\left\{v_{f(\varepsilon)}+S_{\varepsilon}: \varepsilon \in\{-1,1\}^{n}\right\}$ is a collection of simplices spanned by vertices of $P_{C}$. An illustration for $n=2$ is given in Figure 1 .

The following lemma shows how relative interior points of $P_{C}$ are covered by relative interior points of $v_{f(\varepsilon)}+S_{\varepsilon}$ and $v_{f(\varepsilon)}+F_{\varepsilon}$. 

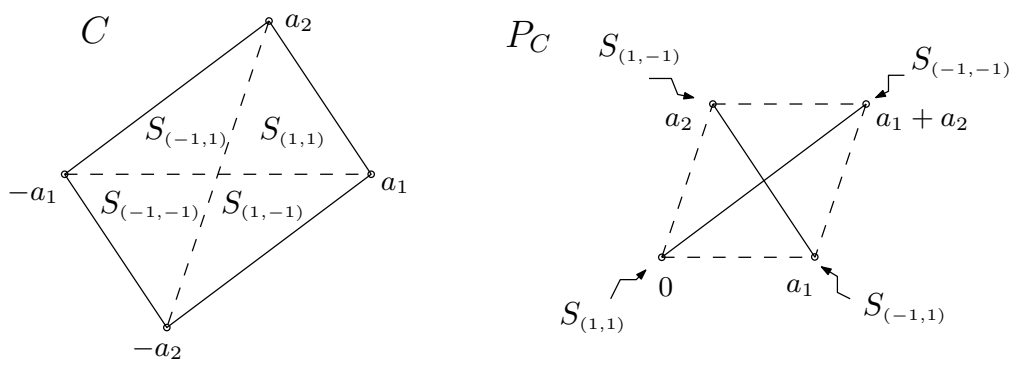

FiguRE 1. Two-dimensional illustration of the dissection of $C$ and its rearrangement in $P_{C}$.

Lemma 3.1. Let $\varepsilon, \varepsilon^{\prime} \in\{-1,1\}^{n}$.

i) If int $\left(v_{f(\varepsilon)}+S_{\varepsilon}\right) \cap \operatorname{int}\left(v_{f\left(\varepsilon^{\prime}\right)}+S_{\varepsilon^{\prime}}\right) \neq \emptyset$, then $\varepsilon$ and $\varepsilon^{\prime}$ differ in at most one coordinate.

ii) If $\operatorname{int}\left(v_{f(\varepsilon)}+S_{\varepsilon}\right) \cap \operatorname{relint}\left(v_{f\left(\varepsilon^{\prime}\right)}+F_{\varepsilon^{\prime}}\right) \neq \emptyset$, then $\varepsilon$ and $\varepsilon^{\prime}$ differ in at most one coordinate.

iii) If relint $\left(v_{f(\varepsilon)}+F_{\varepsilon}\right) \cap \operatorname{relint}\left(v_{f\left(\varepsilon^{\prime}\right)}+F_{\varepsilon^{\prime}}\right) \neq \emptyset$ and $n \geq 3$, then $\varepsilon$ and $\varepsilon^{\prime}$ differ in at most one coordinate. For $n=2$ there is no restriction on $\varepsilon$ and $\varepsilon^{\prime}$.

Proof. We only give the arguments for i), since those for ii) and iii) are analogous. By assumption there is an $x \in \operatorname{int}\left(v_{f(\varepsilon)}+S_{\varepsilon}\right) \cap \operatorname{int}\left(v_{f\left(\varepsilon^{\prime}\right)}+S_{\varepsilon^{\prime}}\right)$. This point has two representations with barycentric coordinates of the vertices of $v_{f(\varepsilon)}+S_{\varepsilon}$ and $v_{f\left(\varepsilon^{\prime}\right)}+S_{\varepsilon^{\prime}}$, respectively. That is, there are $\alpha, \beta \in(0,1)^{n+1}$ with $\sum_{i=0}^{n} \alpha_{i}=\sum_{i=0}^{n} \beta_{i}=1$ such that

$$
\begin{aligned}
x & =\alpha_{0} \sum_{j=1}^{n} \frac{1-\varepsilon_{j}}{2} a_{j}+\sum_{i=1}^{n} \alpha_{i}\left(\sum_{j=1}^{n} \frac{1-\varepsilon_{j}}{2} a_{j}+\varepsilon_{i} a_{i}\right) \\
& =\beta_{0} \sum_{j=1}^{n} \frac{1-\varepsilon_{j}^{\prime}}{2} a_{j}+\sum_{i=1}^{n} \beta_{i}\left(\sum_{j=1}^{n} \frac{1-\varepsilon_{j}^{\prime}}{2} a_{j}+\varepsilon_{i}^{\prime} a_{i}\right) .
\end{aligned}
$$

Collecting $a_{i}$ 's and using $\sum_{i=0}^{n} \alpha_{i}=\sum_{i=0}^{n} \beta_{i}=1$ yields

$$
\sum_{i=1}^{n}\left(\frac{1-\varepsilon_{i}}{2}+\alpha_{i} \varepsilon_{i}\right) a_{i}=\sum_{i=1}^{n}\left(\frac{1-\varepsilon_{i}^{\prime}}{2}+\beta_{i} \varepsilon_{i}^{\prime}\right) a_{i} .
$$

Since the $a_{i}$ 's are linearly independent, these representations coincide and we get $\left(2 \alpha_{i}-1\right) \varepsilon_{i}=\left(2 \beta_{i}-1\right) \varepsilon_{i}^{\prime}$, for all $i=1, \ldots, n$. The coordinates of $\varepsilon$ and $\varepsilon^{\prime}$ are either 1 or -1 , and so $\alpha_{i}=\beta_{i}$ whenever $\varepsilon_{i}=\varepsilon_{i}^{\prime}$, and $\alpha_{j}=1-\beta_{j}$ whenever $\varepsilon_{j}=-\varepsilon_{j}^{\prime}$. Let us relabel the indices such that $\varepsilon_{i}=\varepsilon_{i}^{\prime}$ for $i=1, \ldots, k$ and $\varepsilon_{j}=-\varepsilon_{j}^{\prime}$ for $j=k+1, \ldots, n$. This means that $k$ is the number of coordinates where $\varepsilon$ and $\varepsilon^{\prime}$ agree. Exploiting $\sum_{i=0}^{n} \alpha_{i}=\sum_{i=0}^{n} \beta_{i}=1$ and $\alpha_{i}, \beta_{i}>0$ we 
obtain

$$
\begin{aligned}
1 & =\sum_{i=0}^{n} \alpha_{i}=\alpha_{0}+\sum_{i=1}^{k} \beta_{i}+\sum_{j=k+1}^{n}\left(1-\beta_{j}\right) \\
& =\alpha_{0}+\sum_{i=1}^{k} \beta_{i}-\sum_{j=k+1}^{n} \beta_{j}+n-k=\alpha_{0}+\beta_{0}-1+2 \sum_{i=1}^{k} \beta_{i}+n-k \\
& >n-k-1 .
\end{aligned}
$$

Therefore, $k \geq n-1$ which leaves only one coordinate where $\varepsilon$ and $\varepsilon^{\prime}$ may be different.

Note, that this lemma generalizes for all $i \geq 1$ to $i$-dimensional simplices $v_{f(\varepsilon)}+S_{\delta}$, where $S_{\delta}$ is a face of $S_{\varepsilon}$ and \# $\operatorname{supp}(\delta)=i$, by applying the same argument in a fixed $i$-face of $P_{C}$.

We need a last ingredient for the proof of Theorem 1.3 .

Lemma 3.2. Let $P \in \mathcal{P}^{n}$ be a lattice parallelepiped and write $\mathcal{F}_{i}(P)$ for the union of all $i$-faces of $P, 0 \leq i \leq n$. Then

$$
2^{n} \operatorname{vol}(P)=\sum_{i=0}^{n} 2^{i} \mathrm{G}\left(\operatorname{relint} \mathcal{F}_{i}(P)\right) .
$$

Proof. Since the claimed equality is invariant under lattice translations of $P$, we can assume that $P=\sum_{i=1}^{n}\left[0, v_{i}\right]$, for some linearly independent $v_{1}, \ldots, v_{n} \in \mathbb{Z}^{n}$. It is well-known (e.g. [2, p. 89]) that the volume of $P$ is given by the number of lattice points in its half-open counterpart, that is, $\operatorname{vol}(P)=\#\left(\sum_{i=1}^{n}\left[0, v_{i}\right) \cap \mathbb{Z}^{n}\right)$. At any vertex of $P$ we now place a copy of $P$ which is seen to be generated by the edges that emanate from that vertex. In this way we have counted the lattice points in $P$ with a multiplicity that we can determine. Indeed, the lattice points in the relative interior of an $i$-face $F$ of $P$ are covered exactly $2^{i}$ times, since $F$ has that many vertices and a copy of $P$ contributes relative interior lattice points to $F$ if and only if it was placed at a vertex of $F$. Further, we get $2^{n}$ times the volume of $P$ in this way and the desired formula follows.

Similarly to the definition of $\mathcal{F}_{i}(P)$ in the preceding lemma we write

$$
\mathcal{T}_{i}(C)=\bigcup_{\varepsilon \in\{-1,1\}^{n}} \bigcup_{\delta \in \Delta_{i}(\varepsilon)}\left(v_{f(\varepsilon)}+\stackrel{\circ}{S}_{\delta}\right) .
$$

By construction we have $\mathcal{T}_{i}(C) \subseteq \operatorname{relint} \mathcal{F}_{i}\left(P_{C}\right)$, for all $i=2, \ldots, n$, and $\mathcal{T}_{0}(C)=\operatorname{relint} \mathcal{F}_{0}\left(P_{C}\right)=\operatorname{vert}\left(P_{C}\right)$. Furthermore, $\mathcal{T}_{1}(C)=\operatorname{relint} \mathcal{F}_{1}\left(P_{C}\right) \cup$ relint $\mathcal{F}_{0}\left(P_{C}\right)$.

Proof of Theorem 1.3. The volume of $C$ is given by

$$
\operatorname{vol}(C)=\frac{2^{n}}{n !}\left|\operatorname{det}\left(a_{1}, \ldots, a_{n}\right)\right|=\frac{2^{n}}{n !} \operatorname{vol}\left(P_{C}\right) .
$$


So we can start from Lemma 3.2 and obtain

$$
\begin{aligned}
n ! \operatorname{vol}(C) & =2^{n} \operatorname{vol}\left(P_{C}\right)=\sum_{i=0}^{n} 2^{i} \mathrm{G}\left(\operatorname{relint} \mathcal{F}_{i}\left(P_{C}\right)\right) \\
& \geq \sum_{i=2}^{n} 2^{i} \mathrm{G}\left(\mathcal{T}_{i}(C)\right)+2 \mathrm{G}\left(\operatorname{relint} \mathcal{F}_{1}\left(P_{C}\right)\right)+2^{n} .
\end{aligned}
$$

Lemma 3.1 implies that for all $i \geq 2$, every lattice point in relint $\mathcal{F}_{i}\left(P_{C}\right)$ is covered at most four times by simplices $v_{f(\varepsilon)}+\stackrel{\circ}{S}_{\delta}$ with $\# \operatorname{supp}(\delta)=i$. Therefore, by virtue of Equation (3.1) we get for $i \geq 2$,

$$
\mathrm{G}\left(\mathcal{T}_{i}(C)\right) \geq \frac{1}{4} \sum_{\varepsilon \in\{-1,1\}^{n}} \sum_{\delta \in \Delta_{i}(\varepsilon)} \mathrm{G}\left(\stackrel{\circ}{S}_{\delta}\right)=2^{n-i-2} \mathrm{G}\left(\mathcal{S}_{i}\right) .
$$

Next, we need to consider the lattice points in the edges of $P_{C}$. We have

$$
\begin{aligned}
2 \mathrm{G}\left(\operatorname{relint} \mathcal{F}_{1}\left(P_{C}\right)\right) & =\sum_{\varepsilon \in\{-1,1\}^{n}} \sum_{\delta \in \Delta_{1}(\varepsilon)} \mathrm{G}\left(\operatorname{relint}\left(S_{\delta}\right)\right) \\
& =\sum_{\varepsilon \in\{-1,1\}^{n}} \sum_{\delta \in \Delta_{1}(\varepsilon)}\left(\mathrm{G}\left(\stackrel{\circ}{\delta}_{\delta}\right)-1\right)=2^{n-1} \mathrm{G}\left(\mathcal{S}_{1}\right)-n 2^{n} .
\end{aligned}
$$

With these two relations we can now continue Inequality (3.2) by

$$
\begin{aligned}
n ! \operatorname{vol}(C) & \geq 2^{n-2} \sum_{i=2}^{n} \mathrm{G}\left(\mathcal{S}_{i}\right)+2^{n-1} \mathrm{G}\left(\mathcal{S}_{1}\right)-n 2^{n}+2^{n} \\
& =2^{n-2}\left(\mathrm{G}(C)-\mathrm{G}\left(\mathcal{S}_{1}\right)-\mathrm{G}\left(\mathcal{S}_{0}\right)\right)+2^{n-1} \mathrm{G}\left(\mathcal{S}_{1}\right)-n 2^{n}+2^{n} \\
& \geq 2^{n-2}(\mathrm{G}(C)-(2 n-3)) .
\end{aligned}
$$

The last inequality follows, since by definition of $\stackrel{\circ}{S}_{\delta}$ we have $\mathrm{G}\left(\stackrel{\circ}{S}_{\delta}\right)=1$, for $\# \operatorname{supp}(\delta)=0$, and $\mathrm{G}\left(\stackrel{\circ}{S}_{\delta}\right) \geq 1$, for $\# \operatorname{supp}(\delta)=1$, and therefore $\mathrm{G}\left(\mathcal{S}_{0}\right)=1$ and $\mathrm{G}\left(\mathcal{S}_{1}\right) \geq 2 n$.

\section{LATTICE ZONOTOPES}

Lemma 4.1. Let $P \in \mathcal{P}^{n}$ be a lattice parallelepiped. Then

$$
\operatorname{vol}(P) \geq\left(\frac{1}{2}\right)^{n-1}\left(\mathrm{G}(P)-2^{n-1}\right),
$$

and equality holds if and only if $P \simeq \sum_{i=1}^{n-1}\left[0, e_{i}\right]+\left[0, k e_{n}\right]$, for some $k \in \mathbb{N}$.

Proof. Using the notation from Lemma 3.2 we clearly have

$$
\mathrm{G}(P)=\sum_{i=0}^{n} \mathrm{G}\left(\text { relint } \mathcal{F}_{i}(P)\right) \quad \text { and } \quad \mathrm{G}\left(\text { relint } \mathcal{F}_{0}(P)\right)=2^{n} \text {. }
$$


Thus, Lemma 3.2 yields

$$
\begin{aligned}
\operatorname{vol}(P) & =\sum_{i=0}^{n} 2^{i-n} \mathrm{G}\left(\operatorname{relint} \mathcal{F}_{i}(P)\right) \\
& \geq\left(\frac{1}{2}\right)^{n-1} \sum_{i=1}^{n} \mathrm{G}\left(\operatorname{relint} \mathcal{F}_{i}(P)\right)+1=\left(\frac{1}{2}\right)^{n-1}\left(\mathrm{G}(P)-2^{n-1}\right) .
\end{aligned}
$$

We also see that equality holds if and only if $\mathrm{G}\left(\right.$ relint $\left.\mathcal{F}_{i}(P)\right)=0$, for all $i=2, \ldots, n$, which means that all lattice points of $P$ are contained in its edges. By a suitable lattice translation we can write $P=\sum_{i=1}^{n}\left[0, a_{i}\right]$ for some linearly independent generators $a_{i} \in \mathbb{Z}^{n}$. Then there is at most one non-primitive generator, say $a_{n}$, among the $a_{i}$ 's. Indeed, if we assume $a_{1}$ would also be non-primitive then the interior lattice points in the segments $\left[0, a_{1}\right]$ and $\left[0, a_{n}\right]$ yield an interior lattice point in the 2 -face $\left[0, a_{1}\right]+\left[0, a_{n}\right]$ of $P$ which has just been excluded.

So, there is a $k \in \mathbb{N}$ such that $a_{n}^{\prime}=\frac{1}{k} a_{n} \in \mathbb{Z}^{n}$ is primitive and the halfopen lattice parallelepiped $P^{\prime}=\sum_{i=1}^{n-1}\left[0, a_{i}\right)+\left[0, a_{n}^{\prime}\right)$ contains exactly one lattice point. This means that the generators $a_{1}, \ldots, a_{n-1}, a_{n}^{\prime}$ span a basis of $\mathbb{Z}^{n}$ which gives the equality characterization.

A dissection property of zonotopes allows us to transfer this inequality to the rich class of lattice zonotopes.

Proof of Theorem $1.4 \mathrm{i}$ ). First of all, up to a lattice translation any lattice zonotope has the form $Z=\sum_{i=1}^{m}\left[0, a_{i}\right]$, for some $a_{1}, \ldots, a_{m} \in \mathbb{Z}^{n}$ and $m \geq n$. Analogously to the planar case, Shephard's result [15, Thm. (54)] gives a dissection $\mathcal{C}(Z)$ of $Z$ into parallelepipeds that are lattice translates of $\sum_{j=1}^{n}\left[0, a_{i_{j}}\right]$. Such a dissection can be obtained by starting with $\mathcal{C}_{n}(Z)$ which consists of $\sum_{i=1}^{n}\left[0, a_{i}\right]$ and then successively processing the generators $a_{j}$ for $j>n$ to obtain collections $\mathcal{C}_{j}(Z)$ of parallelepipeds in the following way: for a given generator $a_{j}$ consider all the facets of parallelepipeds of $\mathcal{C}_{j-1}(Z)$ that can be "seen" by $a_{j}$, that is to say that there is a point on the ray $\left\{\lambda a_{j}: \lambda \geq 0\right\}$ that lies beyond the facet. These facets together with the segment $\left[0, a_{j}\right]$ generate the new parallelepipeds in $\mathcal{C}_{j}(Z) \backslash \mathcal{C}_{j-1}(Z)$. After having processed all the generators in this way we let $\mathcal{C}(Z)=\mathcal{C}_{m}(Z)$. This process naturally induces an ordering $P_{1}, \ldots, P_{t}$ of the parallelepipeds in $\mathcal{C}(Z)$ such that for all $1<j \leq t$ the intersection $P_{j} \cap\left(\bigcup_{i=1}^{j-1} P_{i}\right)$ contains at least a facet of $P_{j}$.

In order to prove the claimed inequality for $Z=\bigcup_{i=1}^{t} P_{i}$, we proceed by induction on $t$. The case $t=1$ is precisely Lemma 4.1. So, let $t \geq 2$ and 
write $Q_{t-1}=\bigcup_{i=1}^{t-1} P_{i}$. By the induction hypothesis and Lemma 4.1 we get

$$
\begin{aligned}
\operatorname{vol}(Z) & =\operatorname{vol}\left(P_{t} \cup Q_{t-1}\right)=\operatorname{vol}\left(P_{t}\right)+\operatorname{vol}\left(Q_{t-1}\right) \\
& \geq\left(\frac{1}{2}\right)^{n-1}\left(\mathrm{G}\left(P_{t}\right)-2^{n-1}+\mathrm{G}\left(Q_{t-1}\right)-2^{n-1}\right) \\
& \geq\left(\frac{1}{2}\right)^{n-1}\left(\mathrm{G}(Z)-2^{n-1}\right) .
\end{aligned}
$$

The last inequality holds since as noted above $P_{t} \cap Q_{t-1}$ contains at least a facet of $P_{t}$ and thus at least $2^{n-1}$ lattice points which then are counted twice.

In order to derive the equality case characterization let us assume that $t>1$ and that no two of the generators of $Z$ are parallel (which we can always do). The argumentation above shows that equality can only hold if the intersection $P_{t} \cap Q_{t-1}$ is precisely a facet, say $F$, of $P_{t}$. By construction of $\mathcal{C}(Z)$ there must be some $j \in\{1, \ldots, t-1\}$ such that $P_{t} \cap P_{j}=F$. Now, the zonotope $Z=P_{t} \cup Q_{t-1}$ is convex, which means that $P_{j}$ must be contained in the intersection of the half-spaces corresponding to the facets of $P_{t}$ that are not equal to $F$. But this is a contradiction since the set of generators of $P_{t}$ and $P_{j}$ must be different. Thus, equality can only hold for $t=1$ and by Lemma $4.1 Z$ is unimodularly equivalent to the claimed parallelepiped.

4.1. Lattice zonotopes centrally symmetric to a lattice point. In the following we are concerned with lattice zonotopes whose center of symmetry is also a lattice point. While the inequality in the first part of Theorem 1.4 clearly also holds for these zonotopes, we will show that we can say considerably more if we deal with lattice zonotopes of the form $Z=\sum_{i=1}^{m}\left[-a_{i}, a_{i}\right]$, where $a_{i} \in \Lambda$. Every face of such a lattice zonotope, which by a result of Bolker [6, Thm. 3.3] is centrally symmetric itself, is indeed symmetric to a lattice point of $\Lambda$.

The following lemma could be proved by a more lengthy version of the arguments that were given in the proof of Lemma 4.1. Instead we will present another method of proof that, on the other hand, is also applicable for Lemma 4.1. Its formulation is with respect to an arbitrary lattice $\Lambda \in \mathcal{L}^{n}$ which is necessary for the inductive argument.

Lemma 4.2. Let $\Lambda \in \mathcal{L}^{n}$ be a lattice, $a_{1}, \ldots, a_{n} \in \Lambda$ be linearly independent and consider the parallelepiped $P=\sum_{i=1}^{n}\left[-a_{i}, a_{i}\right]$. Then

$$
\frac{\operatorname{vol}(P)}{\operatorname{det}(\Lambda)} \geq\left(\frac{2}{3}\right)^{n-1}\left(\mathrm{G}(P, \Lambda)-3^{n-1}\right),
$$

and equality holds if and only if $P \simeq \sum_{i=1}^{n-1}\left[-b_{i}, b_{i}\right]+\left[-k b_{n}, k b_{n}\right]$, for some $k \in \mathbb{N}$ and a basis $\left\{b_{1}, \ldots, b_{n}\right\}$ of $\Lambda$.

Proof. In order to use an inductive argument we consider for $l=1, \ldots, n$ the $l$-dimensional lattice parallelepiped $P_{l}=\sum_{i=1}^{l}\left[-a_{i}, a_{i}\right]$ and the sublattice $\Lambda_{l}=\operatorname{lin}\left(P_{l}\right) \cap \Lambda$ of $\Lambda$ that lies in the linear hull of $P_{l}$. For the equality case 
characterization let $R_{k}^{l}=\sum_{i=1}^{l-1}\left[-b_{i}, b_{i}\right]+\left[-k b_{l}, k b_{l}\right]$, for some $k \in \mathbb{N}$ and a basis $\left\{b_{1}, \ldots, b_{l}\right\}$ of $\Lambda_{l}$.

For $l=1$ it is straightforward that $\frac{\operatorname{vol}_{1}\left(P_{1}\right)}{\operatorname{det}\left(\Lambda_{1}\right)}=\mathrm{G}\left(P_{1}, \Lambda_{1}\right)-1$ and that there is a $k \in \mathbb{N}$ such that $P_{1}=R_{k}^{1}$. So let $l \geq 2$. Then, by the geometry of $P_{l}$,

$$
\operatorname{vol}_{l}\left(P_{l}\right)=2\left\|a_{l} \mid \operatorname{lin}\left(P_{l-1}\right)^{\perp}\right\| \operatorname{vol}_{l-1}\left(P_{l-1}\right)
$$

where $a_{l} \mid \operatorname{lin}\left(P_{l-1}\right)^{\perp}$ is the orthogonal projection of $a_{l}$ to the orthogonal complement $\operatorname{lin}\left(P_{l-1}\right)^{\perp}$ of $\operatorname{lin}\left(P_{l-1}\right)$ which is taken in $\operatorname{lin}\left(P_{l}\right)$. Let $w_{l} \in \mathbb{N}$ be such that $2 w_{l}+1$ is the number of lattice hyperplanes in $\Lambda_{l}$ that are parallel to $\Lambda_{l-1}$ and intersect $P_{l}$. Put differently, $2 w_{l}$ is the lattice width of $P_{l}$ with respect to $\Lambda_{l}$ in direction orthogonal to $\operatorname{lin}\left(P_{l-1}\right)$. If we consider $\Lambda_{l-1} \subset \Lambda_{l}$ as a sublattice, we can use a result from lattice theory (see [12, Prop. 1.2.9]) to obtain

$$
\begin{aligned}
\operatorname{det}\left(\Lambda_{l}\right) & =\operatorname{det}\left(\Lambda_{l-1}\right) \cdot \operatorname{det}\left(\Lambda_{l} \mid \operatorname{lin}\left(P_{l-1}\right)^{\perp}\right) \\
& =\operatorname{det}\left(\Lambda_{l-1}\right) \cdot \frac{\left\|a_{l} \mid \operatorname{lin}\left(P_{l-1}\right)^{\perp}\right\|}{w_{l}} .
\end{aligned}
$$

Now by the relations (4.1), 4.2) and the induction hypothesis we get

$$
\begin{aligned}
\frac{\operatorname{vol}_{l}\left(P_{l}\right)}{\operatorname{det}\left(\Lambda_{l}\right)} & =\frac{2\left\|a_{l} \mid \operatorname{lin}\left(P_{l-1}\right)^{\perp}\right\| \operatorname{vol}_{l-1}\left(P_{l-1}\right)}{\operatorname{det}\left(\Lambda_{l-1}\right) \cdot \frac{\left\|a_{l} \mid \operatorname{lin}\left(P_{l-1}\right)^{\perp}\right\|}{w_{l}}} \\
& \geq 2 w_{l}\left(\frac{2}{3}\right)^{l-2}\left(\mathrm{G}\left(P_{l-1}, \Lambda_{l-1}\right)-3^{l-2}\right),
\end{aligned}
$$

which is greater or equal than $\left(\frac{2}{3}\right)^{l-1}\left(\mathrm{G}\left(P_{l}, \Lambda_{l}\right)-3^{l-1}\right)$ if and only if

$$
3 w_{l} \mathrm{G}\left(P_{l-1}, \Lambda_{l-1}\right) \geq \mathrm{G}\left(P_{l}, \Lambda_{l}\right)+3^{l-1}\left(w_{l}-1\right) .
$$

This inequality holds by $\mathrm{G}\left(P_{l-1}, \Lambda_{l-1}\right) \geq 3^{l-1}$ and $\left(2 w_{l}+1\right) \mathrm{G}\left(P_{l-1}, \Lambda_{l-1}\right) \geq$ $\mathrm{G}\left(P_{l}, \Lambda_{l}\right)$, where the latter comes from counting lattice points in $P_{l}$ by the lattice hyperplanes that are parallel to $\Lambda_{l-1}$ and the relation

$$
\mathrm{G}\left(t+P_{l-1}, \Lambda_{l-1}\right) \leq \mathrm{G}\left(P_{l-1}, \Lambda_{l-1}\right) \text {, for all } t \in \operatorname{lin}\left(P_{l-1}\right) .
$$

This last inequality follows by partitioning $P_{l-1}$ into half-open "sub-parallelepipeds" and using the well-known fact that $\mathrm{G}(t+Q) \in\{0, \mathrm{G}(Q)\}$, for any $t \in \mathbb{R}^{n}$, and any possibly lower-dimensional half-open lattice parallelepiped $Q$ (see for instance [8, Sect. 2]).

Equality is attained if and only if there is equality in the induction hypothesis and the equations $\mathrm{G}\left(P_{l-1}, \Lambda_{l-1}\right)=3^{l-1}$ and $\left(2 w_{l}+1\right) \mathrm{G}\left(P_{l-1}, \Lambda_{l-1}\right)=$ $\mathrm{G}\left(P_{l}, \Lambda_{l}\right)$ hold true. This means that the generators $\left\{a_{1}, \ldots, a_{l-1}\right\}$ of $P_{l-1}$ span a basis of $\Lambda_{l-1}$ and $a_{l}$ is an integral multiple of some $b_{l} \in \Lambda_{l}$ which completes that basis to a basis of $\Lambda_{l}$. In other words, $P_{l}$ is unimodularly equivalent to $R_{k}^{l}$, for some $k \in \mathbb{N}$. 
Remark 4.1. For lattice parallelepipeds whose center of symmetry is a lattice point, but which are not of the form $\sum_{i=1}^{n}\left[-a_{i}, a_{i}\right]$, for some $a_{i} \in \mathbb{Z}^{n}$, the inequality in Lemma 4.2 does not hold in general.

As an example consider $P_{k}=\left[-k v_{1}, k v_{1}\right]+\left[-v_{2}, v_{2}\right]+\left[-v_{3}, v_{3}\right]$ with $v_{1}=$ $\left(\frac{1}{2}, \frac{1}{2}, 0\right), v_{2}=\left(\frac{1}{2}, 0, \frac{1}{2}\right), v_{3}=\left(0, \frac{1}{2}, \frac{1}{2}\right) . P_{k}$ is a lattice parallelepiped for odd $k \in \mathbb{N}$, and we have $\operatorname{vol}\left(P_{k}\right)=\frac{2}{5} \cdot \frac{k}{k-1}\left(\mathrm{G}\left(P_{k}\right)-9\right)$.

Examples for any dimension are obtained by successively taking prisms over $P_{k}$. More precisely, for $P_{k}^{n}=P_{k}+\sum_{i=4}^{n}\left[-e_{i}, e_{i}\right]$, we have $\operatorname{vol}\left(P_{k}^{n}\right)=$ $\frac{2}{5}\left(\frac{2}{3}\right)^{n-3} \frac{k}{k-1}\left(\mathrm{G}\left(P_{k}^{n}\right)-3^{n-1}\right)$.

Theorem 1.4 ii) now follows from Lemma 4.2 in the same way as Theorem 1.4 i) followed from Lemma 4.1 .

Acknowledgement. We would like to thank the anonymous referee for helpful comments and suggestions.

\section{REFERENCES}

[1] I. Bárány, Extremal problems for convex lattice polytopes: a survey, Contemp. Math., 453 (2008), Surveys on discrete and computational geometry, 87-103.

[2] A. I. Barvinok, Integer points in polyhedra, Zürich Lectures in Advanced Mathematics, European Mathematical Society (EMS), Zürich, Switzerland, 2008.

[3] M. Beck and S. Robins, Computing the continuous discretely, Undergraduate Texts in Mathematics, Springer, New York, 2007, Integer-point enumeration in polyhedra.

[4] C. Bey, M. Henk, J. M. Wills, Notes on the roots of Ehrhart polynomials, Discrete Comput. Geom., 38 (2007), no. 1, 81-98.

[5] H. F. Blichfeldt, The April meeting of the San Francisco section of the AMS, Amer. Math. Monthly, 28 (1921), no. 6/7, 285-292.

[6] E. D. Bolker, A Class of Convex Bodies, Trans. Amer. Math. Soc., 145 (1969), 323345.

[7] G. Hegedüs, A. M. Kasprzyk, The boundary volume of a lattice polytope, http:// arxiv.org/abs/1002.2815v3.

[8] M. Henk, J. M. Wills, A Blichfeldt-type inequality for the surface area, Mh. Math., 154 (2008), no. 2, 135-144.

[9] T. Hibi, A lower bound theorem for Ehrhart polynomials of convex polytopes, Adv. Math., 105 (1994), no. 2, 162-165.

[10] G. Kalai, The number of faces of centrally-symmetric polytopes, Graphs Combin., 5 (1989), 389-391.

[11] K. Kołodziejczyk, D. Olszewska, A proof of Coleman's conjecture, Discrete Math., 307 (2007), 1865-1872.

[12] J. Martinet, Perfect lattices in Euclidean spaces, Springer, 2003.

[13] G. A. Pick, Geometrisches zur Zahlenlehre, Sitzungsber. Lotus Prag, 19 (1899), 311319.

[14] P. R. Scott, On convex lattice polygons, Bull. Austral. Math. Soc., 15 (1976), 395-399.

[15] G. C. Shephard, Combinatorial properties of associated zonotopes, Can. J. Math., 26 (1974), 302-321.

[16] R. P. Stanley, On the Number of Faces of Centrally-Symmetric Simplicial Polytopes, Graphs Combin., 3 (1987), 55-66.

[17] G. M. Ziegler, Lectures on polytopes, Springer, 1995. 
Fakultät für Mathematik, Otto-von-Guericke Universität Magdeburg, Universitätsplatz 2, D-39106 Magdeburg, Germany

E-mail address: \{martin.henk, matthias .henze\}@ovgu.de

Mathematisches Institut, Universität Siegen, Emmy-Noether-Campus, D57068 Siegen, Germany

E-mail address: wills@mathematik.uni-siegen.de 